\title{
Inhibitory Effect of Synthetic Flavone Derivatives on Pan-Aurora Kinases: Induction of G2/M Cell-Cycle Arrest and Apoptosis in HCT116 Human Colon Cancer Cells
}

\author{
Soon Young Shin ${ }^{1,2}\left(\right.$, , Youngshim Lee ${ }^{3}$, Beom Soo Kim ${ }^{3}$, Junho Lee ${ }^{3}$, Seunghyun Ahn ${ }^{3}$, \\ Dongsoo Koh ${ }^{4}$, Yoongho Lim $^{3}$ and Young Han Lee ${ }^{1,2, *(D)}$ \\ 1 Department of Biological Sciences, Sanghuh College of Life Science s, Konkuk University, Seoul 05029, \\ Korea; shinsy@konkuk.ac.kr \\ 2 Cancer and Metabolism Institute, Konkuk University, Seoul 05029, Korea \\ 3 Division of Bioscience and Biotechnology, BMIC, Konkuk University, Seoul 05029, Korea; \\ librashim@gmail.com (Y.L.); kimbs0812@naver.com (B.S.K.); cttyofjoy@naver.com (J.L.); \\ mistahn321@naver.com (S.A.); yoongho@konkuk.ac.kr (Y.L.) \\ 4 Department of Applied Chemistry, Dongduk Women's University, Seoul 02748, Korea; \\ dskoh@dongduk.ac.kr \\ * Correspondence: yhlee58@konkuk.ac.kr; Tel.: +82-2-2049-6115
}

Received: 28 November 2018; Accepted: 14 December 2018; Published: 17 December 2018

\begin{abstract}
Members of the aurora kinase family are Ser/Thr kinases involved in regulating mitosis. Multiple promising clinical trials to target aurora kinases are in development. To discover flavones showing growth inhibitory effects on cancer cells, 36 flavone derivatives were prepared, and their cytotoxicity was measured using a long-term clonogenic survival assay. Their half-maximal growth inhibitory effects against HCT116 human colon cancer cells were observed at the sub-micromolar level. Pharmacophores were derived based on three-dimensional quantitative structure-activity calculations. Because plant-derived flavones inhibit aurora kinase B, we selected 5-methoxy-2-(2methoxynaphthalen-1-yl)-4H-chromen-4-one (derivative 31), which showed the best half-maximal cell growth inhibitory effect, and tested whether it can inhibit aurora kinases in HCT116 colon cancer cells. We found that derivative $\mathbf{3 1}$ inhibited the phosphorylation of aurora kinases A, aurora kinases B and aurora kinases $C$, suggesting that derivative $\mathbf{3 1}$ is a potential pan-aurora kinase inhibitor. The results of our analysis of the binding modes between derivative $\mathbf{3 1}$ and aurora A and aurora B kinases using in-silico docking were consistent with the pharmacophores proposed in this study.
\end{abstract}

Keywords: flavones; colon cancer; clonogenicity; apoptosis; aurora kinases; quantitative structure-activity relationship; in-silico docking

\section{Introduction}

Flavones are a class of flavonoids having a backbone of 2-phenylchromen-4-one (2-phenyl-1-benzopyran-4-one). They are common in fruits and many plant foods. Some natural flavones show anticancer activity. For example, apigenin $\left(4^{\prime}, 5,7\right.$-trihydroxyflavone), luteolin $\left(3^{\prime}, 4^{\prime}, 5,7-\right.$ tetrahydroxyflavone), baicalein (5,6,7-trihydroxyflavone), nobiletin $\left(3^{\prime}, 4^{\prime}, 5,6,7,8\right.$-hexamethoxyflavone) and tangeretin $\left(4^{\prime}, 5,6,7,8\right.$-pentamethoxyflavone) inhibit the proliferation of breast cancer cells [1-3]. Kaempferol (3,4',5,7-tetrahydroxyflavone) suppresses the growth of bladder and cervical cancer cells [4]. Chrysin (5,7-dihydroxyflavone) reduces the proliferation of prostate cancer cells [5]. Quercetagetin (3,3', 4',5,6,7-hexahydroxyflavone) induces apoptosis in colon cancer cells [6]. However, the structural features of flavones exhibiting inhibitory effects on cancer cell growth remain unclear. 
In order to identify the structural characteristics of flavones exhibiting growth inhibitory effects on cancer cells, we prepared 36 synthetic flavone derivatives containing hydroxy, fluoro, bromo, nitro, methoxy, methyl, styryl or naphthalenyl groups [7]. Colon cancer is the second most diagnosed cancer in women and the third in men [8]. It is also the second most death-causing cancer [8]. Tumor penetration and metastasis can occur in stage II of colon cancer; thus, its survival rate is approximately $70 \%$ after stage II. Even when the resection of colon cancer was achieved by the first treatment of colon cancer owing to diagnosis at the early stage, chemotherapy is often required simultaneously with other treatments [9]. Several drugs have been approved as colon cancer chemotherapy. Drugs as adjuvant chemotherapy after surgery are being developed [10]. Here, we used a long-term clonogenic assay to measure the growth inhibitory effects of synthetic flavones in HCT116 human colon cancer cells. The half-maximal cell growth inhibitory concentration $\left(\mathrm{GI}_{50}\right)$ values of 36 flavone derivatives against HCT116 cells were determined. To derive structural features that exhibit better cell growth inhibitory effects, relationships between the structural properties of the synthetic flavones and their cell growth inhibitory effects were calculated using comparative molecular field analysis and comparative molecular similarity index analysis. Among the 36 synthetic flavone derivatives, we found that 5-methoxy-2-(2-methoxynaphthalen-1-yl)-4H-chromen-4-one (named derivative 31) showed the best $\mathrm{GI}_{50}$ value $(0.49 \mu \mathrm{M})$. Because we have shown in our previous study that plant-derived flavones inhibit aurora kinase B (AURKB) [6], we further evaluated the effect of derivative 31 on the inhibition of aurora kinases. The molecular binding modes between the derivative 31 compound and aurora kinases were elucidated using in-silico docking experiments. These results can provide valuable information for designing novel anticancer drugs that target aurora kinases.

\section{Results and Discussion}

To investigate the structural features of flavones that influence the growth of cancer cells, we synthesized 36 flavone derivatives and screened candidate compounds by monitoring their growth inhibitory efficacy by a long-term clonogenic survival assay [11], which can efficiently distinguish differences in cell growth rate induced by structurally similar compounds [12,13].

Initially, we tested the inhibitory activity of the derivatives at high $(0,5,10,20$ and $40 \mu \mathrm{M})$ (Figure 1A) and low concentrations $(0,0.1,0.5,1$ and $5 \mu \mathrm{M})$ (Figure 1B) on the clonogenicity of cancer cells. Clonogenicity was quantitated by densitometry, $\mathrm{GI}_{50}$ values were computed using the SigmaPlot software, and the results are shown in Table 1 and Figure 2.

Table 1. Names of synthetic flavone derivatives 1-36, and their half-maximal cell growth inhibitory effect $\left(\mathrm{GI}_{50}\right)$ and the negative logarithmic scales of $\mathrm{GI}_{50}$ values $\left(\mathrm{pGI}_{50}\right)$.

\begin{tabular}{|c|c|c|c|}
\hline Derivatives & Chemical Names & $\mathrm{GI}_{50}(\mu \mathrm{M})$ & $\mathrm{pGI}_{50}$ \\
\hline 1 & 2-(2-fluorophenyl)-3-hydroxy-4H-chromen-4-one/2'-fluoroflavone & 41.19 & 1.39 \\
\hline 2 & 2-(2-fluorophenyl)-3-hydroxy-6-nitro-4H-chromen-4-one/2'-fluoro-6-nitroflavone & 33.52 & 1.47 \\
\hline 3 & 2-(4-fluorophenyl)-3-hydroxy-6-nitro-4H-chromen-4-one/4'-fluoro-6-nitroflavone & 4.49 & 2.35 \\
\hline 4 & 3-hydroxy-2-(4-methoxyphenyl)-4H-chromen-4-one/4'-methoxyflavone & 37.18 & 1.43 \\
\hline 5 & 3-hydroxy-2-(2-methoxyphenyl)-4H-chromen-4-one/2'-methoxyflavone & 16.44 & 1.78 \\
\hline 6 & 2-(3,4-dimethoxyphenyl)-3-hydroxy-4H-chromen-4-one $/ 3^{\prime}, 4^{\prime}$-dimethoxyflavone & 3.59 & 2.44 \\
\hline 7 & 3-hydroxy-2-(2,4,6-trimethoxyphenyl)-4H-chromen-4-one $/ 2^{\prime}, 4^{\prime}, 6^{\prime}$-trimethoxyflavone & 4.53 & 2.34 \\
\hline 8 & 2-(2,4-dimethoxyphenyl)-3-hydroxy-4H-chromen-4-one $/ 2^{\prime}, 4^{\prime}$-dimethoxyflavone & 21.92 & 1.66 \\
\hline 9 & $\begin{array}{l}\text { 2-(6-(4-methoxystyryl)-2,4-dimethoxyphenyl)-3-hydroxy-4H-chromen-4-one/3-hydroxy-2'- } \\
\text { (4-methoxystyryl)-flavone }\end{array}$ & 3.18 & 2.50 \\
\hline 10 & $\begin{array}{l}\text { 2-(6-(4-methoxystyryl)-2,4-dimethoxyphenyl)-3-hydroxy-6-nitro-4H-chromen-4-one/3-hydroxy-6- } \\
\text { nitro-2'-(4-methoxystyryl)-flavone }\end{array}$ & 3.63 & 2.44 \\
\hline 11 & $\begin{array}{l}\text { 2-(6-(4-methoxystyryl)-2,4-dimethoxyphenyl)-6-bromo-3-hydroxy-4H-chromen-4-one/3-hydroxy- } \\
\text { 6-bromo-2'-(4-methoxystyryl)-flavone }\end{array}$ & 2.66 & 2.58 \\
\hline 12 & $\begin{array}{l}\text { 2-(6-(4-methoxystyryl)-2,4-dimethoxyphenyl)-7-fluoro-3-hydroxy-4H-chromen-4-one/7-fluoro- } \\
\text { 3-hydroxy-2'-(4-methoxystyryl)-flavone }\end{array}$ & 3.07 & 2.51 \\
\hline 13 & $\begin{array}{l}\text { 2-(6-(4-methoxystyryl)-2,4-dimethoxyphenyl)-6-chloro-3-hydroxy-4H-chromen-4-one/3-hydroxy- } \\
\text { 6-chloro-2'-(4-methoxystyryl)-flavone }\end{array}$ & 2.87 & 2.54 \\
\hline
\end{tabular}


Table 1. Cont

\begin{tabular}{|c|c|c|c|}
\hline Derivatives & Chemical Names & $\mathrm{GI}_{50}(\mu \mathrm{M})$ & $\mathrm{pGI}_{50}$ \\
\hline 14 & $\begin{array}{l}\text { 2-(6-(4-methoxystyryl)-2,4-dimethoxyphenyl)-6-fluoro-3-hydroxy-4H-chromen-4-one/3-hydroxy- } \\
\text { 6-fluoro-2'-(4-methoxystyryl)-flavone }\end{array}$ & 2.41 & 2.62 \\
\hline 15 & 3-hydroxy-2-(naphthalen-1-yl)-4H-chromen-4-one/3-hydroxy-2',3'-naphthoflavone & 4.14 & 2.38 \\
\hline 16 & $\begin{array}{l}\text { 3-hydroxy-6-methoxy-2-(naphthalen-1-yl)-4H-chromen-4-one/3-hydroxy-6-methoxy- } \\
\qquad 2^{\prime}, 3^{\prime} \text {-naphthoflavone }\end{array}$ & 7.21 & 2.14 \\
\hline 17 & $\begin{array}{l}\text { 3-hydroxy-2-(2-methoxynaphthalen-1-yl)-4H-chromen-4-one/3-hydroxy-6'-methoxy- } \\
\text { 2',3'-naphthoflavone }\end{array}$ & 5.28 & 2.28 \\
\hline 18 & $\begin{array}{l}\text { 2-(2,3-dimethoxynaphthalen-1-yl)-3-hydroxy-6-methoxy-4H-chromen-4-one/3-hydroxy-5',6,6' } \\
\text { trimethoxy-2', } 3^{\prime} \text {-naphthoflavone }\end{array}$ & 7.51 & 2.12 \\
\hline 19 & $\begin{array}{l}\text { 3-hydroxy-2-(4-methoxynaphthalen-1-yl)-4H-chromen-4-one/3-hydroxy-4'-methoxy- } \\
\text { 2', }\end{array}$ & 4.28 & 2.37 \\
\hline 20 & 3-hydroxy-2-(naphthalen-2-yl)-4H-chromen-4-one/3-hydroxy-3',4'-naphthoflavone & 2.41 & 2.62 \\
\hline 21 & $\begin{array}{l}\text { 3-hydroxy-6-methoxy-2-(naphthalen-2-yl)-4H-chromen-4-one/3-hydroxy-6-methoxy- } \\
\qquad 3^{\prime}, 4^{\prime} \text {-naphthoflavone }\end{array}$ & 3.07 & 2.51 \\
\hline 22 & 2-(naphthalen-1-yl)-4H-chromen-4-one $/ 2^{\prime}, 3^{\prime}$-naphthoflavone & 2.91 & 2.54 \\
\hline 23 & 6-methoxy-2-(naphthalen-1-yl)-4H-chromen-4-one/6-methoxy-2',3'-naphthoflavone & 2.41 & 2.62 \\
\hline 24 & 5-methoxy-2-(naphthalen-1-yl)-4H-chromen-4-one/5-methoxy-2',3'-naphthoflavone & 7.31 & 2.14 \\
\hline 25 & 6,7-dimethoxy-2-(naphthalen-1-yl)-4H-chromen-4-one/6,7-dimethoxy-2',3'-naphthoflavone & 3.26 & 2.49 \\
\hline 26 & 7-methoxy-2-(naphthalen-1-yl)-4H-chromen-4-one/7-methoxy-2',3'-naphthoflavone & 2.56 & 2.59 \\
\hline 27 & 2-(naphthalen-2-yl)-4H-chromen-4-one $/ 3^{\prime}, 4^{\prime}$-naphthoflavone & 29.86 & 1.52 \\
\hline 28 & 6-methoxy-2-(naphthalen-2-yl)-4H-chromen-4-one/6-methoxy-3', $4^{\prime}$-naphthoflavone & 24.39 & 1.61 \\
\hline 29 & 2-(2-methoxynaphthalen-1-yl)-4H-chromen-4-one $/ 2^{\prime}$-methoxy-2', $3^{\prime}$-naphthoflavone & 4.06 & 2.39 \\
\hline 30 & 6-methoxy-2-(2-methoxynaphthalen-1-yl)-4H-chromen-4-one /2',6-dimethoxy-2', 3'-naphthoflavone & 2.78 & 2.56 \\
\hline 31 & 5-methoxy-2-(2-methoxynaphthalen-1-yl)-4H-chromen-4-one $/ 2^{\prime}, 5$-dimethoxy-2', $3^{\prime}$-naphthoflavone & 0.49 & 3.31 \\
\hline 32 & $\begin{array}{l}\text { 6,7-dimethoxy-2-(2-methoxynaphthalen-1-yl)-4H-chromen-4-one /2',6,7-trimethoxy- } \\
\qquad 2^{\prime}, 3^{\prime} \text {-naphthoflavone }\end{array}$ & 3.80 & 2.42 \\
\hline 33 & 2-(4-methoxynaphthalen-1-yl)-4H-chromen-4-one $/ 4^{\prime}$-methoxy-2',3'-naphthoflavone & 20.80 & 1.68 \\
\hline 34 & $\begin{array}{l}\text { 5,7-dimethoxy-2-(4-methoxynaphthalen-1-yl)-4H-chromen-4-one } / 4^{\prime}, 5,7 \text {-trimethoxy- } \\
\qquad 2^{\prime}, 3^{\prime} \text {-naphthoflavone }\end{array}$ & 21.56 & 1.67 \\
\hline 35 & 7-methoxy-2-(4-methoxynaphthalen-1-yl)-4H-chromen-4-one /4' ,7-dimethoxy-2', $3^{\prime}$-naphthoflavone & 18.18 & 1.74 \\
\hline 36 & $\begin{array}{l}\text { 2-(2,3-dimethoxynaphthalen-1-yl)-7-methoxy-4H-chromen-4-one } / 2^{\prime}, 3^{\prime}, 7 \text {-trimethoxy- } \\
\qquad 2^{\prime}, 3^{\prime} \text {-naphthoflavone }\end{array}$ & 3.95 & 2.40 \\
\hline
\end{tabular}

A

Concentration $(\mu \mathrm{M})$
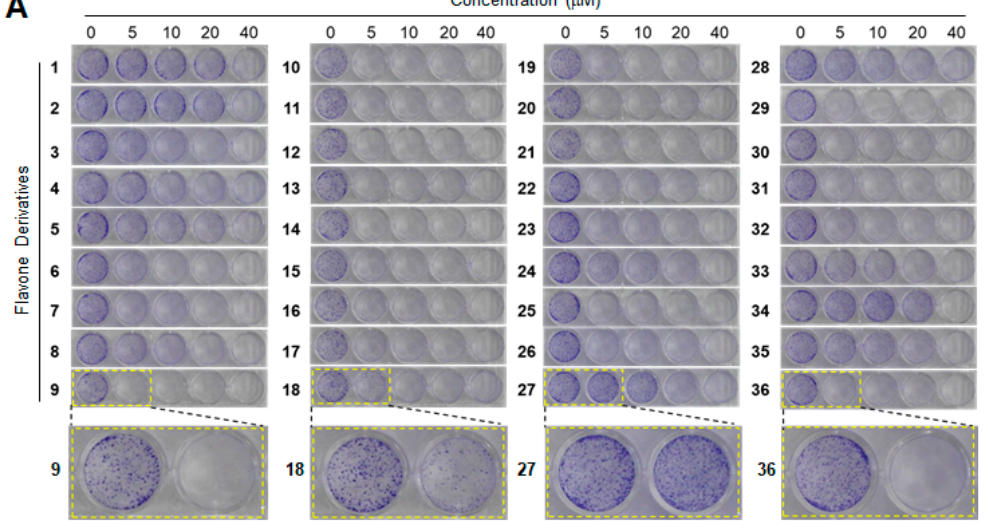

Concentration $(\mu \mathrm{M})$

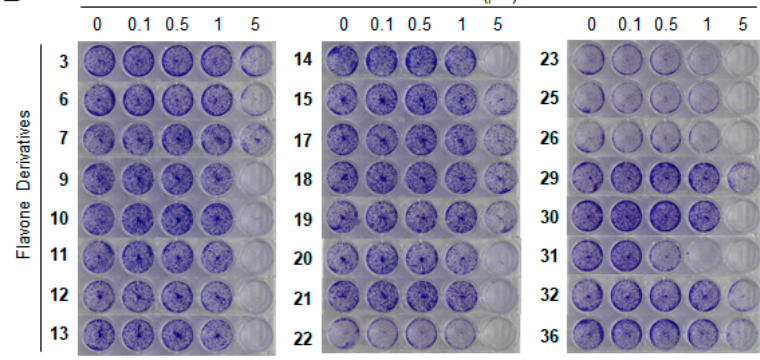

Figure 1. Effects of flavone derivatives on the inhibition of clonogenicity of HCT116 colon cancer cells. Cells were treated with derivative compounds at $0,5,10,20$ and $40 \mu \mathrm{M}(\mathbf{A})$ or at $0,0.1,0.5,1$ and $5 \mu \mathrm{M}$ (B). The dashed lines show the enlarged images. 


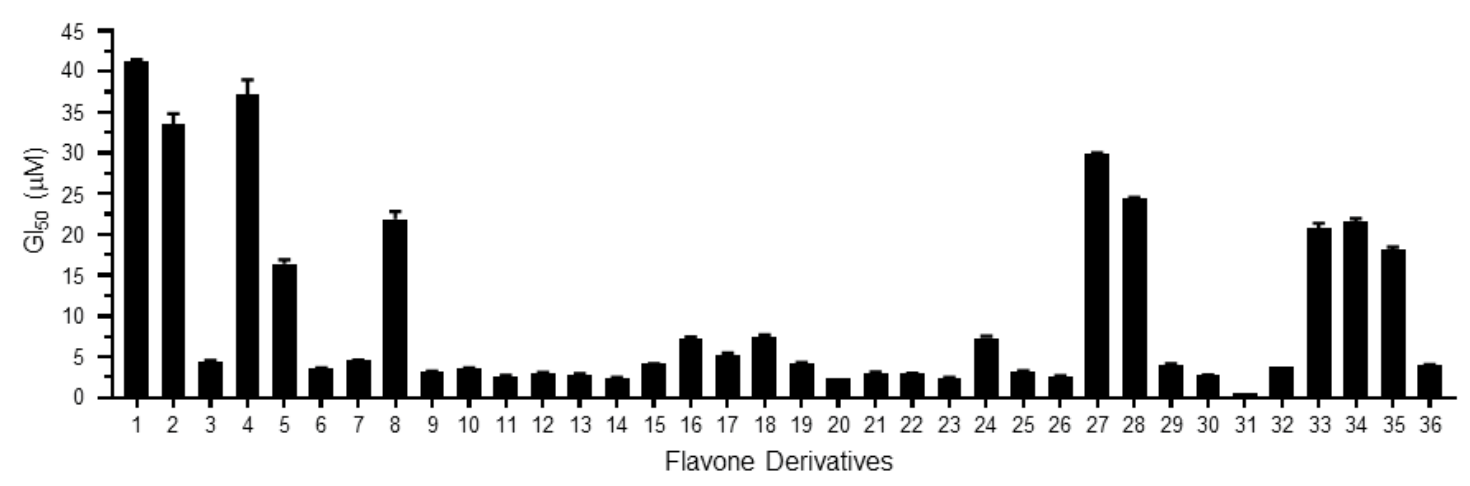

Figure 2. Half-maximal cell growth inhibitory concentration $\left(\mathrm{GI}_{50}\right)$ values in Table 1.

Their $\mathrm{GI}_{50}$ values ranged between 0.49 and $41.19 \mu \mathrm{M}$. Among the derivatives, derivative 31, 5-methoxy-2-(2-methoxynaphthalen-1-yl)-4H-chromen-4-one, showed the most effective inhibition of clonogenicity $\left(\mathrm{GI}_{50}\right.$ value: $0.49 \mu \mathrm{M}$ ). Negative logarithmic scales of the $\mathrm{GI}_{50}$ values (pGI $\mathrm{p}_{50}$ ) were used as biological data for 3D-QSAR calculation. The 3D structures of the derivatives required for 3D-QSAR calculations were modified using the Sybyl 7.3 program from the X-ray crystallographic structures of derivatives 6 (2-(3,4-dimethoxyphenyl)-3-hydroxy-4H-chromen-4-one) and 18 (2-(2,3-dimethoxynaphthalen-1-yl)-3-hydroxy-6-methoxy-4H-chromen-4-one), which were determined by the authors' previous works $[14,15]$. Three-dimensional quantitative structure-activity relationship (3D-QSAR) was performed using comparative molecular field analysis (CoMFA) and comparative molecular similarity indices analysis (CoMSIA). We divided the 36 derivatives into a training set and a test set. The former was used to generate QSAR models and the latter was used to validate the models generated by the training set. Seven derivatives, namely 7, 10, 14, 17, 22, 29 and 32, were arbitrarily chosen for the test set by one of the data analysis tools, hierarchical clustering analysis [16], as shown in Figure S1. Twenty-nine derivatives in the training set were aligned using the Sybyl DATABASE Alignment module. They were aligned well, which indicated interactions between probe atoms and rest atoms (Figure S2). Linear correlations between structural properties of the derivatives in the training set and their cancer cell growth inhibitory effects were determined using partial least-squares regression. Among the many CoMFA models generated by iteration, until a good cross-validation correlation coefficient $\left(\mathrm{q}^{2}\right)$ was found, the model showing 0.772 of $\mathrm{q}^{2}$ was chosen, where non-cross-validated correlation coefficient $\left(\mathrm{r}^{2}\right)$, optimal number of components, standard error of estimate, and F value were $0.980,6,0.078$, and 175.833 , respectively. $\mathrm{pGI}_{50}$ values were predicted based on this model. A comparison of the $\mathrm{pGI}_{50}$ values obtained from the long-term clonogenic survival assay with the values predicted using the CoMFA model is listed in Table S1, and its graph is shown in Figure S3. The residuals between the experimental data and the predicted values ranged from $0.11 \%$ to $8.29 \%$. Likewise, the $\mathrm{pGI}_{50}$ values of the derivatives contained in the test set were calculated using the same CoMFA model (Table S1). The residuals between the experimental data and the predicted values ranged from $6.79 \%$ to $24.96 \%$. These results showed that this CoMFA model was reliable. In this model, the steric and electrostatic field descriptors were $51.7 \%$ and $48.3 \%$, respectively. To visualize the field descriptors, we generated contour maps. The steric-bulk-favoring and -disfavoring regions occupied $92 \%$ and $8 \%$ of the maps, respectively (Figure S4A). In the steric field map of the CoMFA model, green contours were shown at $\mathrm{C}^{\prime}$ and $\mathrm{C}^{\prime}$ positions, meaning that a bulky group was favored in the region. Derivatives containing bulky naphthyl group at the region, such as derivatives 15-18, 22-26, 29-32 and 36, showed good GI 50 values, which were $7.51 \mu \mathrm{M}$ and less. Derivatives $\mathbf{9 - 1 4}$ have the bulky resveratrol group at $\mathrm{C}^{\prime}$ and showed good activities, with $\mathrm{GI}_{50}$ values ranging between $3.63 \mu \mathrm{M}$ and $2.41 \mu \mathrm{M}$. On the contrary, a yellow contour was observed near $\mathrm{C} 4^{\prime}$, meaning that a bulky group was not favored in the region. It is explicable why some of the derivatives containing methoxy group at the $\mathrm{C}^{\prime}$ ' position, such as derivatives $4,8,33,34$ and 35 , showed poor $\mathrm{GI}_{50}$ values. The electrostatic contour map of the CoMFA model is shown in the Figure S4B. The electrostaticly favoring and disfavoring 
regions occupied $1 \%$ and $99 \%$ of the maps, respectively. In the electrostatic field map of the CoMFA model, a small red contour was observed around $\mathrm{C}^{\prime}$. This may be the reason derivatives with fluoride or methoxy substitutes at $\mathrm{C}^{\prime}$ ' showed low biological activities, as shown by derivatives $\mathbf{1}, \mathbf{2}, \mathbf{5}$ and $\mathbf{8}$.

Unlike CoMFA, CoMSIA provides more field descriptors, including hydrogen bond donor and acceptor descriptors and hydrophobic descriptors. To find the best CoMSIA model, many CoMSIA models were generated by iteration. The CoMSIA model showing $\mathrm{q}^{2}$ of 0.515 was selected, where the $\mathrm{r}^{2}$, optimal number of components, standard error of estimate, and $\mathrm{F}$ value were $0.952,6,0.119$, and 73.084, respectively. This model consisted of steric, electrostatic and hydrophobic descriptors, and their contributions were $18.3 \%, 53.3 \%$ and $28.4 \%$, respectively. The pGI $_{50}$ values calculated using this model were compared with those obtained from the experimental values (Table S2), and they were graphed as shown in Figure S5. The residuals between the experimental data and predicted values ranged from $0.18 \%$ to $10.62 \%$. Likewise, the $\mathrm{pGI}_{50}$ values of derivatives contained in the test set were calculated using the same CoMFA model (Table S2). The residuals between the experimental data and predicted values ranged from $7.34 \%$ to $23.92 \%$. These results showed that this CoMSIA model was reliable. The steric and electrostatic field descriptors were generated to be similar to those obtained from the CoMFA model. A hydrophobic field map (Figure S6) was additionally obtained from the CoMSIA model. The hydrophobic region occupied $95 \%$ of the contour map area, whereas the nonhydrophobic region occupied $5 \%$. The orange-colored contour around $\mathrm{C}^{\prime}$ suggested that a hydrophobic group was favored at the position. The derivatives 33-35 had a methoxy substituent at the $\mathrm{C} 4^{\prime}$ of thier naphthyl moiety and showed lower activities compared to derivatives 22-26, which had naphthyl without a hydrophilic substituent. The pharmacophores obtained based on the CoMFA and CoMSIA models are summarized in Figure 3, which provides insights for rational design of compounds with good inhibitory effects on cell growth. In summary, a bulky group was favored at C2' and C3' but was not favored at $\mathrm{C} 4^{\prime}$. A hydrophobic group was favored at $\mathrm{C} 4^{\prime}$, and an electronegative group was not favored at $\mathrm{C}^{\prime}$. One of the criteria for the solubility is $\log \mathrm{P}$. The $\log \mathrm{P}$ values of derivatives containing a 3-hydroxy group ranged between 1.53 and 3.02, those of derivatives with a styryl group, 3.72 and 5.45, and $\log P$ values of naphthoflavones ranged from 3.69 to 4.07 .

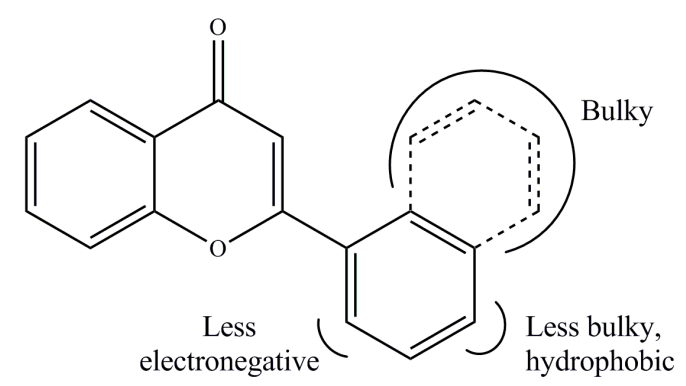

Figure 3. Pharmacophores derived based on the CoMFA and CoMSIA models.

Aurora kinases are Ser/Thr kinases that function as key regulators of chromosome alignment and segregation during mitosis [17]. There are three classes of aurora kinases: aurora kinase A (AURKA), aurora kinase B (AURKB) and aurora kinase C (AURKC). Previously, we showed that plant-derived flavones inhibit AURKB [6]. To investigate whether synthetic flavone derivatives inhibit aurora kinase activity, we selected one of the compounds, derivative 31, which exhibited the best $\mathrm{GI}_{50}$ value, and examined its inhibitory activity against aurora kinases. aurora kinase activity was assessed by its phosphorylation status, as reported previously [18]. Treatment with derivative 31 decreased the phosphorylation of AURKA on Thr-288, AURKB on Thr-232, and AURKC on Thr-198 in a dose- (Figure $4 \mathrm{~A}$ ) and time-dependent (Figure 4B) manner, suggesting that derivative 31 exhibited pan-aurora kinase inhibitory activity. 
A

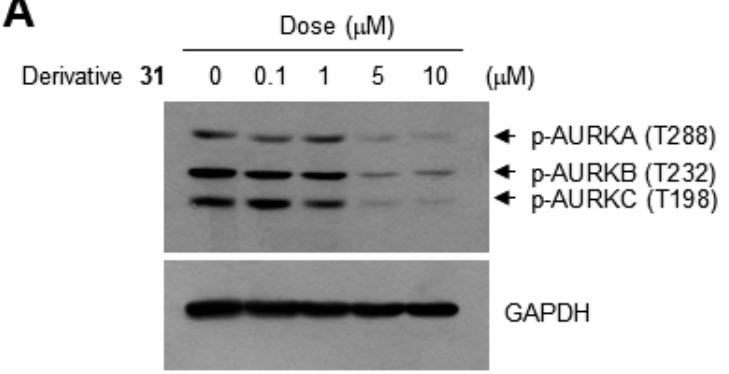

B

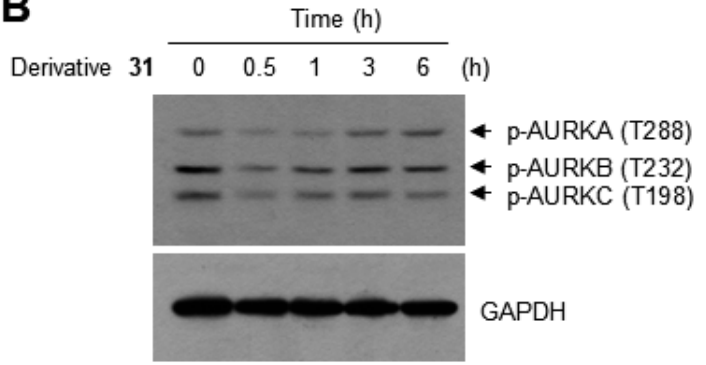

Figure 4. Effect of derivative 31 on inhibition of aurora kinases. HCT116 cells were serum-starved for $24 \mathrm{~h}$ in media containing $0.5 \%$ FBS, and treated with different concentrations of derivative $31(0,0.1,1$, 5 or $10 \mu \mathrm{M})$ for $3 \mathrm{~h}(\mathbf{A})$ or $5 \mu \mathrm{M}$ derivative 31 for different times $(1.0 .5,1,3$ or $6 \mathrm{~h})(\mathbf{B})$. Total cell lysates were immunoblotted with phospho-specific antibodies against AURKA (T288), AURKB (T232) and AURKC (T198). Anti-GAPDH antibody was used as an internal control.

AURKA and AURKB are overexpressed in colon cancer [19], and inhibition of aurora kinases triggers mitotic cell-cycle arrest and apoptotic cell death [18]. Therefore, we investigated by flow cytometry whether derivative 31 affects cell cycle progression. After treatment with derivative 31, population of G2/M phase cells increased from $26.2 \%(0 \mathrm{~h})$ to $43.3 \%(12 \mathrm{~h})$ and $46.3 \%(24 \mathrm{~h}$ ) (Figure $5 \mathrm{~A}$ ). Notably, the number of sub-G1 phase cells remarkably increased from 3\% (0 h) to $31.5 \%(48 \mathrm{~h})$ as the number of G1 phase cells concomitantly decreased from 59.9\% ( $0 \mathrm{~h}$ ) to 8.0\% (48 h) (Figure 5B). Because the presence of sub-G1 cell population is indicative of the progression of apoptotic cells, we suggest that derivative $\mathbf{3 1}$ induced cell-cycle arrest at the G2/M phase at the early stage but triggered apoptotic cell death in HCT116 colon cancer cells after continued exposure. We thus evaluated the capability of derivative 31 to induce apoptosis.

A

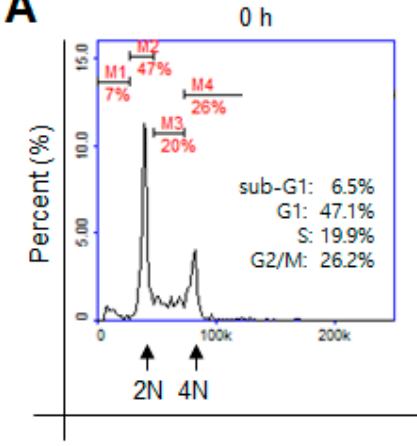

B

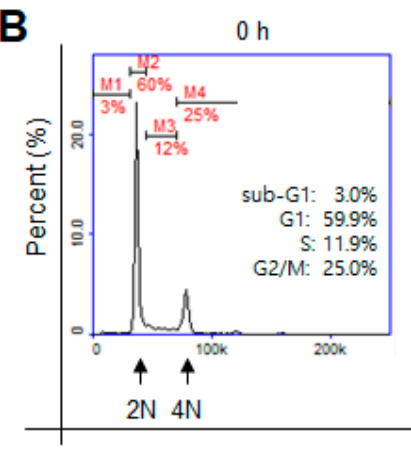

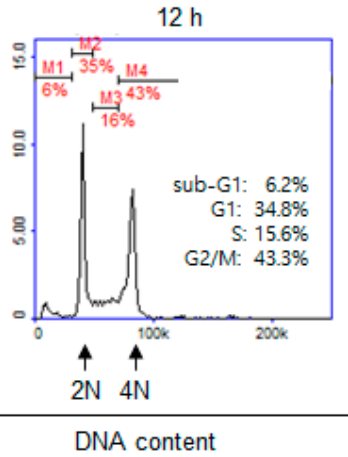

$24 \mathrm{~h}$

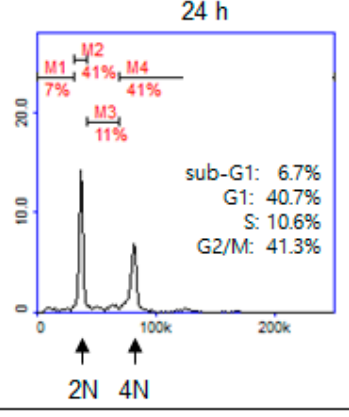

DNA content
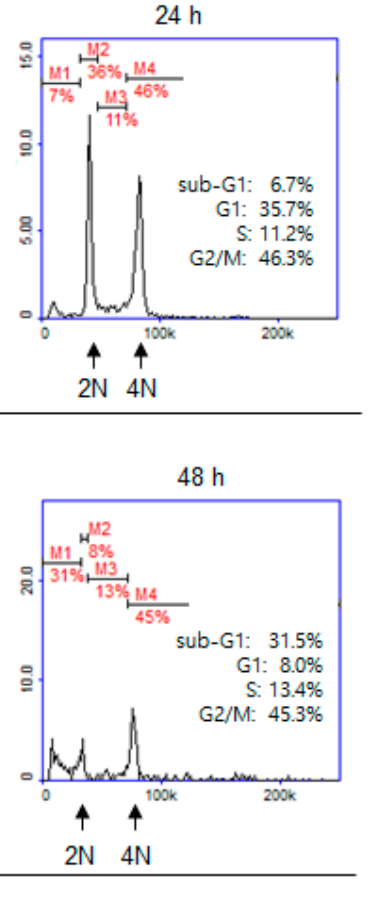

Figure 5. Effect of derivative 31 on G2/M arrest and apoptosis. HCT116 cells were treated with $5 \mu \mathrm{M}$ derivative 31 for 0,12 and $24 \mathrm{~h}$ (A) or 0, 24 and $48 \mathrm{~h}$ (B). The cells were fixed with ethanol and stained with propidium iodide (PI). Cellular DNA contents were determined by flow cytometry. $2 \mathrm{~N}$, diploid; 4N, tetraploid; M1, sub-G1; M2, G1; M2, S, M4, G2/M. 
Because the phosphatidylserine localized in the inner surface of the cell membrane translocates to the outer membrane during apoptosis [20], we analyzed the population of apoptotic cells by staining the outer layer of the cell membrane with phosphatidylserine by using annexin V [21]. Propidium iodide was used as a counterstain to label dead cells. Flow cytometry results showed that treatment with derivative 31 at 5 and $10 \mu \mathrm{M}$ increased the population of annexin V-positive cells from $8 \%$ to $25 \%$ and $73 \%$, respectively (Figure $6 \mathrm{~A}$ ). These data suggested that derivative 31 caused apoptotic cell death in HCT116 cells. Caspases regulate the cleavage of many cellular proteins, including the DNA repair enzyme poly(ADP-ribose) polymerase (PARP), to induce apoptosis [22]. Caspases are activated by proteolytic cleavages [23]. To determine whether derivative 31-induced apoptosis is mediated by caspases, we examined the status of caspase 7 cleavage by Western blotting analysis. We found that the cleavages of caspase 7 and its substrate, PARP, were increased by treatment with derivative $\mathbf{3 1}$ in a time-dependent manner (Figure 6B). Taken together, derivative 31 triggered apoptosis by inhibiting aurora kinases through G2/M cell-cycle arrest and a caspase-dependent mechanism.

$\mathbf{A}$
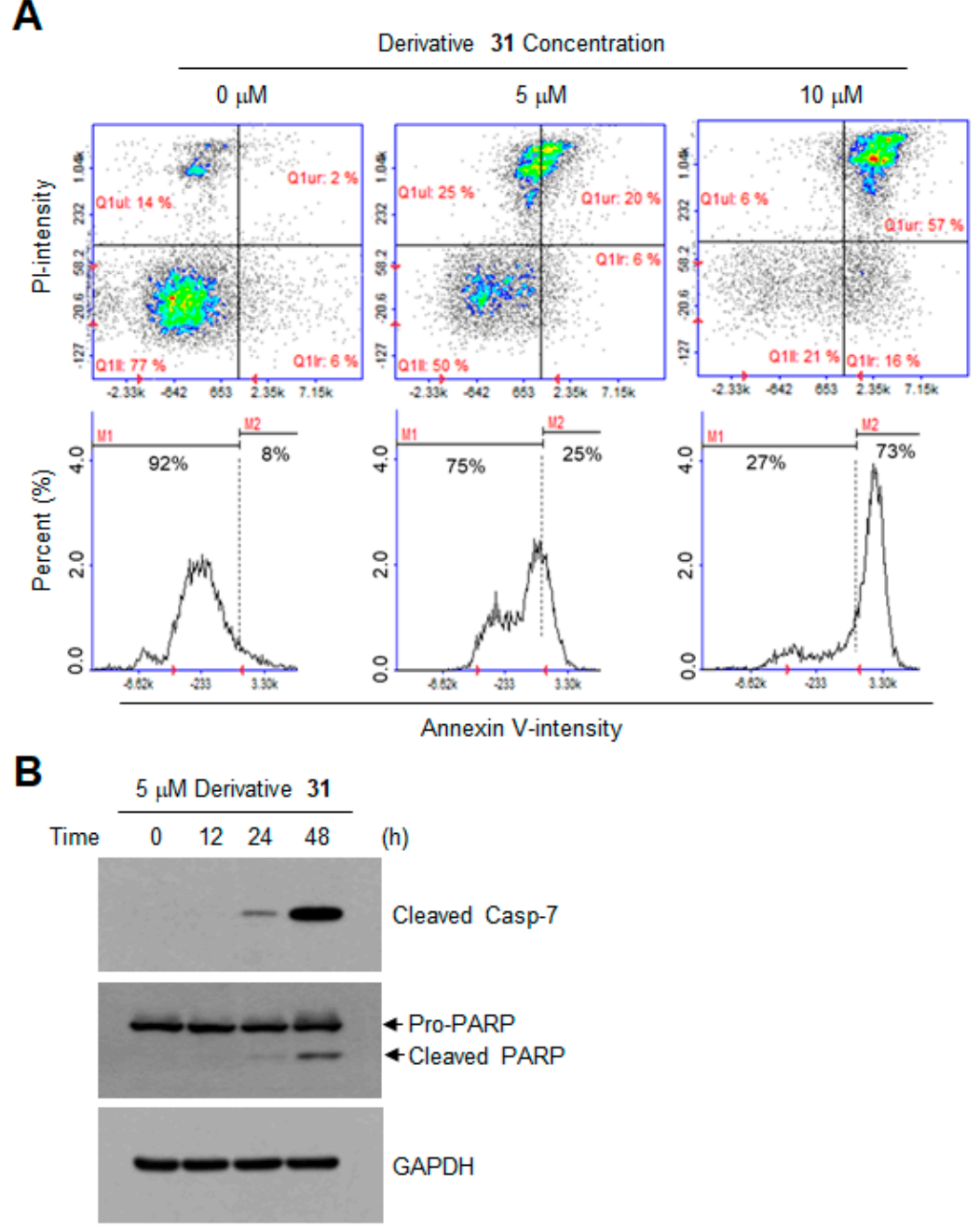

Figure 6. Effect of derivative 31 on apoptosis induction. (A) HCT116 cells were treated with derivative 31 at 0,5 and $10 \mu \mathrm{M}$ for $48 \mathrm{~h}$, and co-stained with fluorescein isothiocyanate (FITC)-annexin V and PI. Fluorescence intensity was analyzed by a NucleoCounter NC-3000 image cytometer. Scatter plots represent FITC-annexin V versus PI intensities (upper panels). Lower graphs represent populations of annexin V-positive cells. M1, annexin V-negative; M2, annexin V-positive. (B) HCT116 cells were treated with $5 \mu \mathrm{M}$ derivative $\mathbf{3 1}$ for $0,6,12$ and $48 \mathrm{~h}$, and total cell lysates were immunoblotted with antibodies against cleaved-caspase-7 and poly(ADP-ribose) polymerase (PARP). Anti-GAPDH antibody was used as an internal control. 
To elucidate the binding modes between derivative $\mathbf{3 1}$ and aurora kinases at the molecular level, in-silico docking experiments were conducted. Among the many X-ray crystallographic structures of AURKA deposited in the protein data bank, 3uod.pdb was selected because its ligand, 4-[(4-[2-(trifluoromethyl)phenyl]amino]pyrimidin-2-yl)amino]benzoic acid (named as TPB) (Figure S7), is more similar to the synthetic flavones used here than the ligands contained in other crystallographic structures deposited in the protein data bank [24]. Its organism, expression system and resolution were Homo sapiens, Escherichia coli BL21(DE3) and $2.5 \AA$, respectively. AURKA consists of 403 residues and 3uod.pdb contains residues between Ser123-Lys401, including a kinase domain. This structure contains its ligand as well as 1,2-ethanediol and di(hydroxyethyl)ether. To prepare the apoprotein of 3uod.pdb, its ligand was extracted using the Sybyl/Biopolymer module (Tripos), but 1,2-ethanediol and di(hydroxyethyl)ether were not deleted. The solution structure of the apoprotein was obtained through energy minimization using the Conjugate Gradient algorithm where Tripos force field and Gasteiger-Hückell charges were used. Because comparing this apoprotein with 3uod.pdb resulted in a root-mean-squared deviation value of $0.7 \AA$, this apoprotein was used for in-silico docking experiments. As mentioned above, the 3D structure of the title compound was determined based on the X-ray crystallographic structure of derivative 18 (2-(2,3-dimethoxynaphthalen-1-yl)-3-hydroxy-6-methoxy-4H-chromen-4-one) [15]. The results obtained from AutoDock Vina were visualized using PyMol (The PyMOL Molecular Graphics System, Version 1.0r1; Schrödinger, LLC), and analyzed using LigPlot [25]. The binding pocket of AURKA was analyzed using the Ligplot software, and 14 residues were obtained: Arg137, Leu139, Gly140, Val147, Ala160, Leu194, Glu211, Tyr212, Ala213, Thr217, Arg220, Glu260, Leu263 and Ala213 (Figure S8). The dimensions of the docking box were 16,8 and 16 for $\mathrm{x}, \mathrm{y}$ and $\mathrm{z}$, respectively, whereas the centers of $x, y$ and $z$ were $21.494,-21.987$ and -10.808 , respectively. Because the flexible docking procedure was iterated 30 times, 30 AURKA apoprotein-ligand complexes were generated. Because the original ligand, TPB, was docked into the apoprotein well, in-silico docking of derivative $\mathbf{3 1}$ was performed in the same manner as that of the original ligand. Because its binding energies ranged from -9.1 to -7.2 $\mathrm{kcal} / \mathrm{mol}$, the thermodynamic stability of the docking process of derivative 31 was considered good for further analysis. The complex with the lowest binding energy was selected. The residues residing in its binding pocket were analyzed using LigPlot: Arg137, Leu139, Val147, Ala160, Leu194, Leu210, Glu211, Tyr212, Ala213, Gly216, Thr217, Arg220, Glu260, Leu263 and Asp274 (Figure S9). The binding pocket was visualized using the PyMol program as shown in Figure 7. Even the AURKA-derivative 31 complex included one more residue in its binding site than the AURKA-TPB complex, and it does not contain hydrogen bonds, unlike the AURKA-TPB complex where two residues, Arg137 and Ala213, participated in hydrogen bonds. The binding pocket around the naphthalene ring of derivative 31 consisted of mainly hydrophobic residues, Val147, Leu210 and Leu263, and the binding pocket was deep and wide enough to hold a naphthyl or resveratrol group. It is also well explained that a bulky group is not favored at the $\mathrm{C}^{\prime}$ position because the side chain of Tyr 212 could induce steric hindrance with substrates. The results of our analysis of the binding mode of derivative $\mathbf{3 1}$ are consistent with the pharmacophores that we proposed.

Because Western blotting analysis showed that treatment with derivative $\mathbf{3 1}$ decreased the phosphorylation of AURKB, the binding mode between derivative 31 and AURKB was elucidated using in-silico docking in the same manner as that of AURKA. Because 4af3.pdb contained the most residues, it was used for in-silico docking [26]. It originated from $H$. sapiens and was expressed in an E. coli BL21 (DE3) system. Its ligand was cyclopropanecarboxylic acid 4-[4-(4-methyl-piperazin1-yl)-6-(5-methyl-2h-pyrazol-3-ylamino)-pyrimidin-2-ylsulfanyl]-phenyl]-amide (named as VX6). The binding pocket of AURKB was analyzed using Ligplot: Leu83, Phe88, Val91, Ala104, Lys106, Leu138, Glu155, Tyr156, Ala157, Gly160, Glu161, Leu207, Ala217, Asp218 and Phe219 (Figure S10). The dimensions and centers of the docking box were the same as those in the AURKA docking condition. Because the original ligand, VX6, was docked into the apoprotein well, in-silico docking of derivative 31 was performed in the same manner as that of the original ligand. The binding energies 
of 30 AURKB-derivative 31 complexes ranged from -9.6 to $-7.8 \mathrm{kcal} / \mathrm{mol}$, which showed that the complexes were thermodynamically stable. The complex with the lowest binding energy was selected. The residues residing in the binding pocket of the complex were analyzed using LigPlot: Leu83, Phe88, Va191, Ala104, Lys106, Glu155, Tyr156, Ala157, Glu161, Glu204, Asn205, Leu207, Ala217 and Phe219 (Figure S11). The binding pocket was visualized using the PyMol program as shown in Figure 8.

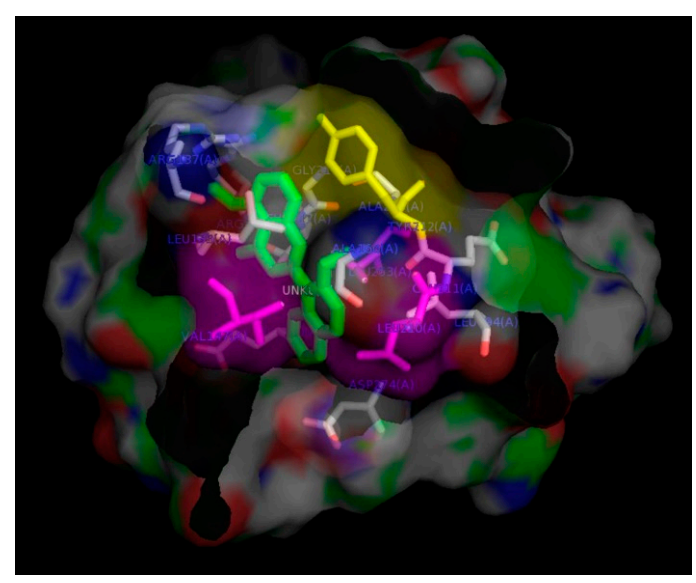

Figure 7. Image of the binding pocket of the AURKA-derivative 31 complex visualized using the PyMol program. Derivative 31 and Tyr212 are colored in green and yellow, respectively. Val147, Leu210 and Leu263 are marked in magenta color.

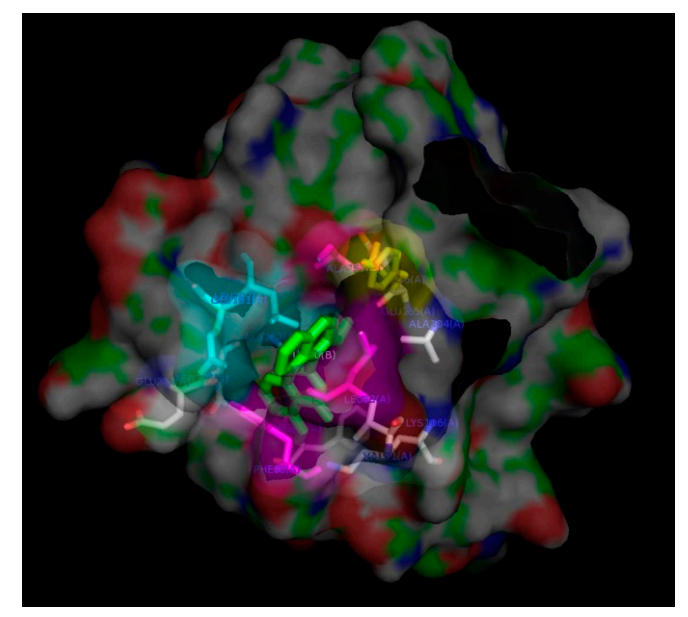

Figure 8. Image of the binding pocket of the AURKB-derivative 31 complex visualized using the PyMol program. Derivative 31 and Tyr156 are colored in green and yellow, respectively. Leu83, Phe88, Ala157 and Leu207 are marked in magenta color. Glu161 is marked in cyan color.

The AURKB-derivative 31 complex contained fewer residues in its binding pocket than the AURKB-VX6 complex. In addition, the AURK-VX6 complex included two hydrogen bonds at Lys106 and Glu155, whereas the AURKB-derivative 31 complex consisted of only hydrophobic interactions. Like the AURKA-derivative $\mathbf{3 1}$ complex, the naphthalenyl group is surrounded by hydrophobic residues, Leu83, Phe88, Ala157 and Leu207, and the side chain of Tyr156 resides in the pocket near the naphthalenyl group. However, the hydrophilic residue Glu161 was near the same pocket; thus, the docking of derivative $\mathbf{3 1}$ was not favored compared to that of AURKA. The results of Western blotting analysis showed that even though derivative 31 decreased the phosphorylation of both AURKA and AURKB in a dose- and time-dependent manner, the binding modes of derivative 31 to AURKA and AURKB at the molecular level were different from each other.

In conclusion, 36 synthetic flavone derivatives at micromolar concentrations showed half-maximal cell growth inhibitory effects against HCT116 human colon cancer cells. The structural conditions that 
showed good inhibitory effects on the growth of colon cancer cells were derived based on 3D-QSAR calculations, including the CoMFA and CoMSIA methods, where a bulky group was favored at $\mathrm{C} 2{ }^{\prime}$ and $\mathrm{C}^{\prime}$ but was not favored at $\mathrm{C} 4^{\prime \prime}$, a hydrophobic group was favored at $\mathrm{C} 4^{\prime}$, and an electronegative group was not favored at $\mathrm{C}^{\prime}$. In our previous study, a flavone derivative inhibited AURKB; thus, Western blotting analysis was performed on derivative 31, which showed the best half-maximal inhibitory effect on cell growth. Because treatment with derivative $\mathbf{3 1}$ decreased the phosphorylation of AURKA, AURKB and AURKC in a dose- and time-dependent manner, this derivative was considered to exhibit pan-aurora kinase inhibitory activity. In addition, flow cytometry results showed that derivative 31 induced apoptosis, and annexin $\mathrm{V}$ staining results showed that it triggered apoptosis by inhibiting aurora kinases through G2/M cell-cycle arrest and a caspase-dependent mechanism. The results of binding mode analysis between derivative 31 and AURKA and AURKB at the molecular level using in-silico docking were consistent with the pharmacophores that we proposed. As a result, the synthetic flavone studied here can be developed as a pan-aurora kinase inhibitor and a chemotherapeutic agent.

\section{Materials and Methods}

\subsection{Preparation of 36 Synthetic Flavone Derivatives}

The synthesis and identification of flavone derivatives containing hydroxy, fluoro, bromo, nitro, methoxy, methyl, styryl, and/or naphthalenyl groups were reported previously [7]. The synthetic scheme is provided as Scheme S1 [7]. The names of the derivatives are listed in Table 1. Infrared (IR) spectra were collected using an FT-IR 4200 spectrophotometer (JASCO, Easton, MD, USA) with attenuated total reflection (ATR PR0450-S). IR data as well as the melting points, yields, and purities are provided in the Supplementary Materials. The structures of the derivatives are provided as Table S3. IR spectra are provided in the Supplementary Materials.

\subsection{Cell Culture}

HCT116 human colon cancer cells were obtained from the American Type Culture Collection (Rockville, MD, USA). The cells were maintained in Dulbecco's modified Eagle's medium supplemented with $10 \%$ fetal bovine serum (CellGro/Corning, Manassas, VA, USA) at $37{ }^{\circ} \mathrm{C}$ in a $5 \% \mathrm{CO}_{2}$ atmosphere [27].

\subsection{Clonogenic Long-Term Survival Assay}

A long-term clonogenic assay was conducted as described previously [11]. Cells were treated with flavone derivatives $(0,5,10,20$, and $40 \mu \mathrm{M})$ for six days. At seven days after treatment, the cells were stained with $0.1 \%$ crystal violet. Among the 36 derivatives, 24 derivatives inhibited the growth of the cancer cells almost completely; thus their clonogenicities were measured at lower concentrations $(0$, $0.1,0.5,1$, and $5 \mu \mathrm{M})$. The inhibitory activities of flavone derivatives on clonogenicity were measured using densitometry (MultiGuage, Fujifilm, Japan), and $\mathrm{GI}_{50}$ values were computed using the SigmaPlot software (version 12, SYSTAT, Chicago, IL, USA) [28].

\subsection{Quantitative Structure-Activity Relationship (QSAR)}

Three-dimensional quantitative structure-activity relationship (3D-QSAR) analysis was performed on an Intel Core 2 Quad Q6600 (2.4 GHz) Linux PC with the Sybyl 7.3 software (Tripos, St. Louis, MO, USA) using comparative molecular field analysis (CoMFA) and comparative molecular similarity indices analysis (CoMSIA). The experiment followed previously reported methods [12].

\subsection{Cell-Cycle Analysis by Flow Cytometry}

Cell-cycle status was examined by flow cytometry using propidium iodide [29]. Briefly, HCT116 cells were treated with $5 \mu \mathrm{M}$ derivative 31 for 0,12 , and $24 \mathrm{~h}$, and fixed in $70 \%(v / v)$ ethanol. Next, the cells were stained with $50 \mu \mathrm{g} / \mathrm{mL}$ propidium iodide solution containing $0.1 \%(v / v)$ Triton X-100, 
$0.1 \mathrm{mM}$ EDTA, and $50 \mu \mathrm{g} / \mathrm{mL}$ RNase A. Cellular DNA contents were detected by a NucleoCounter NC-3000 cytometer (ChemoMetec, Allerød, Denmark). Diploid (2N) and tetraploid (4N) cells represented cells at the G1 and G2/M phases, respectively. $2 \mathrm{~N}$ and $4 \mathrm{~N}$ cells corresponded to those at the $\mathrm{S}$ phase. Cells containing DNA lower than 2N DNA were considered as cells at the sub-G1 phase [30].

\subsection{Apoptosis Assay by Annexin V Staining}

Apoptosis assay was performed using a fluorescein isothiocyanate (FITC)-conjugated annexin V kit (BD Pharmingen, San Diego, CA, USA) according to the manufacturer's instructions. Fluorescence was counted using a NucleoCounter NC-3000 image cytometer (ChemoMetec, Allerød, Denmark) [31].

\subsection{Western Blotting Analysis}

HCT116 cells were treated with derivative 31 for the indicated times. Cell lysates were prepared and immunoblotted according to standard procedures. Antibody-reactive protein bands were visualized using an enhanced chemiluminescence detection system (GE Healthcare, Piscataway, NJ, USA). Antibodies against phospho-aurora kinase A (T288)/aurora kinase B (T232)/aurora kinase C (T198), cleaved caspase-7 (Asp198), and poly(ADP-ribose) polymerase (PARP) were obtained from Cell Signaling Technology (Beverly, MA, USA). Antibodies specific to GAPDH were obtained from Santa Cruz Biotechnology (Santa Cruz, CA, USA) [6].

\subsection{In-Silico Docking}

To elucidate the molecular binding modes between the title compound and aurora kinases, in-silico docking was conducted using AutoDock Vina. In addition, preparation of holoproteins and apoproteins, as well as determination of binding site were performed using the Sybyl program (Tripos) [32]. The 3D structures of aurora kinases were obtained from the protein databank. The experiments followed previously reported methods [27].

\subsection{Statistical Analysis}

Statistical significance was analyzed using Student's $t$-test [6]. A $p$-value of less than 0.05 was considered statistically significant. All experiments were performed in triplicate.

Supplementary Materials: Supplementary materials can be found at http:/ / www.mdpi.com/1422-0067/19/12/ 4086/s1.

Author Contributions: Y.L. (Yoongho Lim), D.K., and Y.H.L. conceived and designed the experiments; S.Y.S., Y.L. (Youngshim Lee), B.S.K., J.L. and S.A. performed experiments and analyzed data; Y.L. (Yoongho Lim) and D.K. contributed preparation of materials; S.Y.S. wrote the paper; Y.H.L and Y.L. (Yoongho Lim) edited the writing; Y.H.L. supervised the project.

Acknowledgments: This work was supported by the Konkuk University Research Support Program in 2015.

Conflicts of Interest: The authors declare no conflict of interest.

\section{Abbreviations}

$\begin{array}{ll}\text { GI }_{50} & \text { cell growth inhibitory concentration } \\ \text { AURKA } & \text { aurora kinase A } \\ \text { AURKB } & \text { aurora kinase B } \\ \text { AURKC } & \text { aurora kinase C } \\ \text { CoMFA } & \text { comparative molecular field analysis } \\ \text { CoMSIA } & \text { comparative molecular similarity indices analysis } \\ \text { 3D-QSAR } & \text { Three-dimensional quantitative structure-activity relationship } \\ \text { PARP } & \text { poly(ADP-ribose) polymerase } \\ \text { TPB } & \text { 4-[(4-[2-(trifluoromethyl)phenyl]amino]pyrimidin-2-yl)amino]benzoic acid } \\ \text { FITC } & \text { fluorescein isothiocyanate }\end{array}$ 


\section{References}

1. Lin, C.H.; Chang, C.Y.; Lee, K.R.; Lin, H.J.; Chen, T.H.; Wan, L. Flavones inhibit breast cancer proliferation through the Akt/FOXO3a signaling pathway. BMC Cancer 2015, 15, 958. [CrossRef] [PubMed]

2. Gade, S.; Gandhi, N.M. Baicalein Inhibits MCF-7 Cell Proliferation In Vitro, Induces Radiosensitivity, and Inhibits Hypoxia Inducible Factor. J. Environ. Pathol. Toxicol. Oncol. 2015, 34, 299-308. [CrossRef] [PubMed]

3. Surichan, S.; Arroo, R.R.; Ruparelia, K.; Tsatsakis, A.M.; Androutsopoulos, V.P. Nobiletin bioactivation in MDA-MB-468 breast cancer cells by cytochrome P450 CYP1 enzymes. Food Chem. Toxicol. 2018, 113, $228-235$. [CrossRef] [PubMed]

4. Dang, Q.; Song, W.; Xu, D.; Ma, Y.; Li, F.; Zeng, J.; Zhu, G.; Wang, X.; Chang, L.S.; He, D.; et al. Kaempferol suppresses bladder cancer tumor growth by inhibiting cell proliferation and inducing apoptosis. Mol. Carcinog. 2015, 54, 831-840. [CrossRef] [PubMed]

5. Samarghandian, S.; Afshari, J.T.; Davoodi, S. Chrysin reduces proliferation and induces apoptosis in the human prostate cancer cell line pc-3. Clinics 2011, 66, 1073-1079. [CrossRef] [PubMed]

6. Jung, Y.; Shin, S.Y.; Yong, Y.; Jung, H.; Ahn, S.; Lee, Y.H.; Lim, Y. Plant-derived flavones as inhibitors of aurora B kinase and their quantitative structure-activity relationships. Chem. Biol. Drug Des. 2015, 85, 574-585. [CrossRef] [PubMed]

7. Kim, B.S.; Koh, E.J.; Jung, Y.; Shin, S.Y.; Lim, Y. ${ }^{1} \mathrm{H}$ and ${ }^{13} \mathrm{C}$ NMR spectral assignments of flavone derivatives. Magn. Reson. Chem. 2017, 55, 359-366. [CrossRef]

8. Meyers, B.M.; Cosby, R.; Quereshy, F.; Jonker, D. Adjuvant Chemotherapy for Stage II and III Colon Cancer Following Complete Resection: A Cancer Care Ontario Systematic Review. Clin. Oncol. 2017, 29, 459-465. [CrossRef]

9. Kannarkatt, J.; Joseph, J.; Kurniali, P.C.; Al-Janadi, A.; Hrinczenko, B. Adjuvant Chemotherapy for Stage II Colon Cancer: A Clinical Dilemma. J. Oncol. Pract. 2017, 13, 233-241. [CrossRef]

10. Tsuji, Y.; Sugihara, K. Adjuvant chemotherapy for colon cancer: The difference between Japanese and western strategies. Expert. Opin. Pharmacother. 2016, 17, 783-790. [CrossRef]

11. Franken, N.A.; Rodermond, H.M.; Stap, J.; Haveman, J.; van Bree, C. Clonogenic assay of cells in vitro. Nat. Protoc. 2006, 1, 2315-2319. [CrossRef] [PubMed]

12. Kim, B.S.; Shin, S.Y.; Ahn, S.; Koh, D.; Lee, Y.H.; Lim, Y. Biological evaluation of 2-pyrazolinyl-1carbothioamide derivatives against HCT116 human colorectal cancer cell lines and elucidation on QSAR and molecular binding modes. Bioorg. Med. Chem. 2017, 25, 5423-5432. [CrossRef] [PubMed]

13. Jung, K.Y.; Park, J.; Han, Y.S.; Lee, Y.H.; Shin, S.Y.; Lim, Y. Synthesis and biological evaluation of hesperetin derivatives as agents inducing apoptosis. Bioorg. Med. Chem. 2017, 25, 397-407. [CrossRef] [PubMed]

14. Yoo, J.S.; Lim, Y.; Koh, D. Crystal structure of 2-(3,4-di-meth-oxy-phen-yl)-3-hy-droxy-4H-chromen-4-one. Acta Crystallogr. Sect. E Struct. Rep. Online 2014, 70. [CrossRef]

15. Ahn, S.; Lim, Y.; Koh, D. Crystal structure of 2-(2,3-di-meth-oxy-naphthalen-1-yl)-3-hy-droxy-6-meth-oxy-4Hchromen-4-one. Acta Crystallogr. E Crystallogr. Commun. 2015, 71. [CrossRef]

16. He, W.; Zhang, M.G.; Wang, X.J.; Zhong, S.; Shao, Y.; Zhu, Y.; Shen, Z.J. AURKA suppression induces DU145 apoptosis and sensitizes DU145 to docetaxel treatment. Am. J. Transl. Res. 2013, 5, 359-367.

17. Nigg, E.A. Mitotic kinases as regulators of cell division and its checkpoints. Nat. Rev. Mol. Cell Biol. 2001, 2, 21-32. [CrossRef]

18. Walter, A.O.; Seghezzi, W.; Korver, W.; Sheung, J.; Lees, E. The mitotic serine/threonine kinase Aurora2/AIK is regulated by phosphorylation and degradation. Oncogene 2000, 19, 4906-4916. [CrossRef]

19. Bischoff, J.R.; Anderson, L.; Zhu, Y.; Mossie, K.; Ng, L.; Souza, B.; Schryver, B.; Flanagan, P.; Clairvoyant, F.; Ginther, C.; et al. A homologue of Drosophila aurora kinase is oncogenic and amplified in human colorectal cancers. EMBO J. 1998, 17, 3052-3065. [CrossRef] [PubMed]

20. Lum, J.J.; Bauer, D.E.; Kong, M.; Harris, M.H.; Li, C.; Lindsten, T.; Thompson, C.B. Growth factor regulation of autophagy and cell survival in the absence of apoptosis. Cell 2005, 120, 237-248. [CrossRef]

21. Andree, H.A.; Reutelingsperger, C.P.; Hauptmann, R.; Hemker, H.C.; Hermens, W.T.; Willems, G.M. Binding of vascular anticoagulant alpha (VAC alpha) to planar phospholipid bilayers. J. Biol. Chem. 1990, 265, 4923-4928. [PubMed]

22. McIlwain, D.R.; Berger, T.; Mak, T.W. Caspase functions in cell death and disease. Cold Spring Harb Perspect. Biol. 2013, 5. [CrossRef] [PubMed] 
23. Riedl, S.J.; Shi, Y. Molecular mechanisms of caspase regulation during apoptosis. Nat. Rev. Mol. Cell Biol. 2004, 5, 897-907. [CrossRef] [PubMed]

24. Martin, M.P.; Zhu, J.Y.; Lawrence, H.R.; Pireddu, R.; Luo, Y.; Alam, R.; Ozcan, S.; Sebti, S.M.; Lawrence, N.J.; Schonbrunn, E. A novel mechanism by which small molecule inhibitors induce the DFG flip in Aurora A. ACS Chem. Biol. 2012, 7, 698-706. [CrossRef]

25. Kramer, B.; Rarey, M.; Lengauer, T. Evaluation of the FLEXX incremental construction algorithm for protein-ligand docking. Proteins 1999, 37, 228-241. [CrossRef]

26. Elkins, J.M.; Santaguida, S.; Musacchio, A.; Knapp, S. Crystal structure of human aurora B in complex with INCENP and VX-680. J. Med. Chem. 2012, 55, 7841-7848. [CrossRef] [PubMed]

27. Lee, Y.; Kim, B.S.; Ahn, S.; Koh, D.; Lee, Y.H.; Shin, S.Y.; Lim, Y. Anticancer and structure-activity relationship evaluation of 3-(naphthalen-2-yl)-N,5-diphenyl-pyrazoline-1-carbothioamide analogs of chalcone. Bioorg. Chem. 2016, 68, 166-176. [CrossRef]

28. Shin, S.Y.; Jung, H.; Ahn, S.; Hwang, D.; Yoon, H.; Hyun, J.; Yong, Y.; Cho, H.J.; Koh, D.; Lee, Y.H.; et al. Polyphenols bearing cinnamaldehyde scaffold showing cell growth inhibitory effects on the cisplatin-resistant A2780/Cis ovarian cancer cells. Bioorg. Med. Chem. 2014, 22, 1809-1820. [CrossRef]

29. Lee, D.H.; Kim, C.G.; Lim, Y.; Shin, S.Y. Aurora kinase A inhibitor TCS7010 demonstrates pro-apoptotic effect through the unfolded protein response pathway in HCT116 colon cancer cells. Oncol. Lett. 2017, 14, 6571-6577. [CrossRef]

30. Shin, S.Y.; Ahn, S.; Yoon, H.; Jung, H.; Jung, Y.; Koh, D.; Lee, Y.H.; Lim, Y. Colorectal anticancer activities of polymethoxylated 3-naphthyl-5-phenylpyrazoline-carbothioamides. Bioorg. Med. Chem. Lett. 2016, 26, 4301-4309. [CrossRef]

31. Gil, H.N.; Koh, D.; Lim, Y.; Lee, Y.H.; Shin, S.Y. The synthetic chalcone derivative 2-hydroxy-3' $5,5^{\prime}-$ trimethoxychalcone induces unfolded protein response-mediated apoptosis in A549 lung cancer cells. Bioorg. Med. Chem. Lett. 2018, 28, 2969-2975. [CrossRef] [PubMed]

32. Trott, O.; Olson, A.J. AutoDock Vina: Improving the speed and accuracy of docking with a new scoring function, efficient optimization, and multithreading. J. Comput. Chem. 2010, 31, 455-461. [CrossRef] [PubMed] 